\begin{tabular}{|c|l|}
\hline Title & Vector fields near a generic submanifold \\
\hline Author(s) & Ishikawa, G.; Izumiya, S.; Watanabe, K. \\
\hline Citation & Hokkaido University Preprint Series in Mathematics, 127, 2-9 \\
\hline Issue Date & 1991-10 \\
\hline DOI & 10.14943/83272 \\
\hline Doc URL & http://hdl.handle.net/2115/68874 \\
\hline Type & bulletin (article) \\
\hline File Information & pre127.pdf \\
\hline
\end{tabular}

Instructions for use 
Vector fields near a generic submanifold

G. Ishikawa, S. Izumiya and K. Watanabe

Series $\sharp 127$. October 1991 


\section{HOKKAIDO UNIVERSITY \\ PREPRINT SERIES IN MATHEMATICS}

$\sharp 100$ : S. Izumiya, Geometric singularities for Hamilton-Jacobi equation, 13 pages. 1991.

$\sharp 101$ : S. Izumiya, Legendrian singularities and first order differential equations, 16 pages. 1991.

\#102: A. Munemasa, Y. Watatani, Orthogonal pairs of *-subalgebras and association schemes, 11 pages. 1991.

$\sharp 103: \quad$ A. Arai, O. Ogurisu, Meromorphic $N=2$ Wess-Zumino supersymmetric quantum mechanics, 27 pages. 1991.

$\sharp 104: \quad$ H. Takamura, Global existence of classical solutions to nonlinear wave equations with spherical symmetry for small data with noncompact support in three space dimensions, 14 pages. 1991.

$\sharp 105$ : R. Agemi, Blow-up of solutions to nonlinear wave equations in two space dimensions, 11 pages. 1991.

$\sharp 106: \quad$ T. Nakazi, Extremal problems in $H^{p}, 13$ pages. 1991.

$\# 107$ : T. Nakazi, $\rho$-dilations and hypo-Dirichlet algebras, 15 pages. 1991.

$\sharp 108$ : A. Arai, An abstract sum formula and its applications to special functions, 25 pages. 1991 .

$\sharp 109$ : Y.-G. Chen, Y. Giga and S. Goto, Analysis toward snow crystal growth, 18 pages. 1991.

$\sharp 110$ : T. Hibi, M. Wakayama, A $q$-analogue of Capelli's identity for $G L(2), 7$ pages. 1991.

\#111: T. Nishimori, A qualitative theory of similarity pseudogroups and an analogy of Sacksteder's theorem, 13 pages. 1991.

$\sharp 112$ : K. Matsuda, An analogy of the theorem of Hector and Duminy, 10 pages. 1991.

$\sharp 113$ : S. Takahashi, On a regularity criterion wo to the boundary for weak solutions of the Navier-Stokes equations, 23 pages. 1991.

$\sharp 114: \quad$ T. Nakazi, Sum of two inner functions and exposed points in $H^{1}, 18$ pages. 1991.

\#115: A. Arai, De Rham operators, Laplacians, and Dirac operators on topological vector spaces, 27 pages. 1991.

$\sharp 116$ : T. Nishimori, A note on the classification of non-singular flows with transverse similarity structures, 17 pages. 1991.

\#117: T. Hibi, A lower bound theorem for Ehrhart polynomials of convex polytopes, 6 pages. 1991.

\# 118: R. Agemi, H. Takamura, The lifespan of classical solutions to nonlinear wave equations in two space dimensions, 30 pages. 1991.

$\sharp 119$ : S. Altschuler, S. Angenent and Y. Giga, Generalized motion by mean curvature for surfaces of rotation, 15 pages. 1991.

$\sharp 120$ : T. Nakazi, Invariant subspaces in the bidisc and commutators, 20 pages. 1991.

$\sharp 121$ : A. Arai, Commutation properties of the partial isometries associated with anticommuting self-adjoint operators, 25 pages. 1991.

$\sharp 122: \quad$ Y.-G. Chen, Blow-up solutions to a finite difference analogue of $u_{t}=\Delta u+u^{1+\alpha}$ in $N$-dimensional balls, 31 pages. 1991.

\# 123: A. Arai, Fock-space representations of the relativistic supersymmetry algebra in the two-dimensional spacetime, 13 pages. 1991.

$\sharp 124$ : S. Izumiya, The theory of Legendrian unfoldings and first order differential equations, 16 pages. 1991.

$\sharp 125$ : T. Hibi, Face number inequalities for matroid complexes and Cohen-Macaulay types of Stanley-Reisner rings of distributive lattices, 17 pages. 1991.

\#126: S. Izumiya, Completely integrable holonomic systems of first order differential equations, 35 pages. 1991. 


\title{
Vector fields near a generic submanifold
}

\author{
Goo Ishikawa, Shyūichi IzumiYa and Kazuo Watanabe
}

\begin{abstract}
Abstruct
It is given a classification of generic vector fields near a generic submanifold. The normal forms are linear vector fields near the local model of the submanifold. Similar results are obtained for vector fields near a hypersurface with boundary and near a piecewise-smooth hypersurface.
\end{abstract}

AMS 1980 Mathematics Subject Classification. Primary 58C27; Secondary 58G20.

\section{Introduction}

In this paper we shall study generic local normal forms of vector fields near a generic submanifold. This classification problem arises naturally in setting up a "directional derivative problem" (cf. V.I. Arnold [1]).

Let $M$ be a smooth $n$-manifold, $Q$ a smooth $(n-k)$-manifold and $\operatorname{Emb}(Q, M)$ the space of smooth embeddings of $Q$ in $M$ endowed with the Whitney topology. We also consider vector fields on $M$. By the Thom's transversality theorem, a generic vector field has only isolated singularities. Thus we may assume that a vector field $X$ on $M$ has only isolated singularities. One of our results is as follows:

THEOREM A. There exists a residual subset $\mathcal{O} \subset \operatorname{Emb}(Q, M)$ with the following property: For any $i \in \mathcal{O}$ and $q \in Q$, there exist a coordinate neighborhood $\left(U,\left(x_{1}, \ldots, x_{n}\right)\right)$ around $i(q)$ in $M$ and an integer $t=t(q),\left(0 \leq t \leq \frac{n}{k}-1\right)$, such that

$$
\begin{aligned}
& \text { (1) } X \mid U=\sum_{j=1}^{t}\left(\sum_{i=1}^{k} x_{j k+i} \frac{\partial}{\partial x_{(j-1) k+i}}\right)+\frac{\partial}{\partial x_{t k+1}}, \\
& \text { (2) } i(Q) \cap U=\left\{\left(x_{1}, \ldots, x_{n}\right) \mid x_{1}=\cdots=x_{k}=0\right\} .
\end{aligned}
$$

We remark that the same normal forms have been obtained by S.M.Vishik [6] in the case when $k=1$. Our assertion and the method of the proof are, however, slightly different from those of him. 
We observe that if $n<2 k$ then the generic normal form of vector fields near a generic submanifold is the trivial vector field $\partial / \partial x_{1}$.

In $\S 1$ we shall prepare a kind of transversality theorem which describes some generic properties of embeddings. The local properties of embeddings will be studied in §2.

In $\S 3$ we will prove Theorem $A$. The main idea of the proof is as follows: Since $X$ has only isolated singularities, we may assume that $X$ is non-singular near the image of a generic embedding. Then $X$ is transformed to $\partial / \partial x_{1}$ by a local diffeomorphism on $M$. Moreover, the image is the zero set of a local submersion. In the first place, we shall classify zero sets of local submersions by diffeomorphisms which preserve the vector field $\partial / \partial x_{1}$. After this procedure, we shall transform local submersions and vector fields to corresponding normal forms in Theorem A by appropriate diffeomorphisms.

In $\S 4$, we shall state other results. We treat the same problem in the case of a generic submanifold with boundary in Theorem B. We also study the case of a generic piecewise smooth hypersurface in Theorem C. Since we can prove Theorems B and C by the same method as that used to show Theorem A, we shall omit details of the proofs of Theorems $\mathrm{B}$ and $\mathrm{C}$.

The multi-germ version of the method we used here can be applied to study shadows of submanifolds in a Euclidian space (K.Watanabe [7]).

All map germs considered here, are differentiable of class $C^{\infty}$, unless stated otherwise.

\section{A transversality theorem}

Since the vector field $X$ has only isolated singularities, there exists a residual set $\mathcal{O}_{1} \subset$ $\operatorname{Emb}(Q, M)$ such that $X$ is non-singular at every points of $i(Q)$ for any $i \in \mathcal{O}_{1}$.

Let $i: Q \rightarrow M$ be an embedding. For any $q \in Q$, we may suppose locally $Q$ is the zero set of a submersion germ $F:(M, i(q)) \rightarrow\left(\mathrm{R}^{k}, 0\right)$. We call $F$ a local equation of $i(Q)$ at $i(q)$. Of course, the choice of $F$ is not unique. It depends only on the $\mathcal{C}$-equivalence in the sense of J.Mather [5]. Thus we use notions and results of the theory on the $\mathcal{K}$-equivalence (cf. J.Mather [5] and J.Martinet [4]).

Let $\mathcal{S}$ be a $\mathcal{K}$-invariant Whitney stratification of the fiber $J^{r}(1, k)$ of the $r$-jet space $J^{r}\left(\mathbf{R}, \mathbf{R}^{k}\right)$. We define a stratification $\tilde{\mathcal{S}}$ of the $r$-jet space by

$$
\left\{\mathbf{R} \times\left\{\mathbf{R}^{k}-\{0\}\right\} \times J^{r}(1, k), \mathbf{R} \times\{0\} \times S \mid S \in \mathcal{S}\right\},
$$

where $J^{r}\left(\mathrm{R}, \mathrm{R}^{k}\right)=\mathrm{R} \times \mathrm{R}^{k} \times J^{r}(1, k)$. Our transversality theorem is as follows:

THEOREM 1.1. There exists a residual set $\mathcal{O} \subset \operatorname{Emb}(Q, M)$ with the following properties: For any $i \in \mathcal{O}$ and $q \in Q$, there exists a local coordinate $\left(x_{1}, \ldots, x_{n}\right)$ of $M$ near $i(q)$ such 
that $X=\partial / \partial x_{1}$ and the germ $j_{1}^{r} F:(M, i(q)) \rightarrow J^{1}\left(\mathbf{R}, \mathbf{R}^{k}\right)$ is transverse to $\tilde{\mathcal{S}}$. Here $F$ is a local equation of $i(Q)$ at $i(q), j_{1}^{r} F\left(x_{1}, \ldots, x_{n}\right)=j^{r} F_{\left(x_{2}, \ldots, x_{n}\right)}\left(x_{1}\right)$ and $F_{\left(x_{2}, \ldots, x_{n}\right)}\left(x_{1}\right)=$ $F\left(x_{1}, \ldots, x_{n}\right)$.

Proof: Let $\Sigma_{X}$ be the singular set of $X$. For any $p \in M-\Sigma_{X}$, there exists a coordinate neighborhood $\left(U,\left(x_{1}, \ldots, x_{n}\right)\right)$ around $p$ with $X=\partial / \partial x_{1}$. We fix countably many such coordinate neighborhoods $U_{\ell},(\ell \in \mathrm{N})$, of $M-\Sigma_{X}$.

Let $J^{r} E(Q, M)$ be the open subset of $J^{r}(Q, M)$ consisting of $r$-jets of local embeddings. For each $\mathbf{d}=\left(d_{1}, \ldots, d_{k}\right)$, with $1<d_{1}<\cdots<d_{k} \leq n$, and for each $\ell \in \mathbf{N}$, define an open subset $\mathcal{R}_{\mathrm{d}, \ell}$ of $J^{r} E(Q, M)$ as follows: $j^{r} i(q) \in \mathcal{R}_{\mathrm{d}, \ell}$ if and only if $i(q) \in U_{\ell}$ and $\operatorname{det}\left(\partial x_{j} \circ i\right) /\left(\partial u_{s}\right)(q) \neq 0$ for $j \neq d_{1}, \ldots, d_{k}, 1 \leq s \leq n-k$, where $\left(u_{1}, \ldots, u_{n-k}\right)$ is a local coordinate of $Q$ around $q$.

For any $j^{r} i(q) \in \mathcal{R}_{\mathrm{d}, \ell}$, we may choose

$$
x^{\mathrm{d}}=\left(x_{1}, \ldots, x_{d_{1}-1}, x_{d_{1}+1}, \ldots, x_{d_{k}-1}, x_{d_{k}+1}, \ldots, x_{n}\right),
$$

as a local coordinate of $Q$ around $q$. With respect to this local coordinate, a representative of $j^{r} i(q)$ is given by

$$
\tilde{i}\left(x^{\mathrm{d}}\right)=\left(x_{1}, \ldots, x_{d_{1}-1}, g_{d_{1}}\left(x^{\mathrm{d}}\right), x_{d_{1}+1}, \ldots, x_{d_{k}-1}, g_{d_{k}}\left(x^{\mathrm{d}}\right), x_{d_{k}+1}, \ldots, x_{n}\right) .
$$

We now define a map-germ

$$
F^{\mathrm{d}, \ell}:\left(\mathrm{R}^{n}, i(q)\right) \rightarrow\left(\mathrm{R}^{k}, 0\right)
$$

by

$$
F^{\mathrm{d}, \ell}=\left(x_{d_{1}}-g_{d_{1}}\left(x^{\mathrm{d}}\right), \ldots, x_{d_{k}}-g_{d_{k}}\left(x^{\mathrm{d}}\right)\right)
$$

Then we have

$$
\left(F^{\mathrm{d}, \ell}\right)^{-1}(0)=\operatorname{Im} \tilde{i}
$$

We also define a surjective submersion

$$
L_{\mathrm{d}, \ell}: \mathcal{R}_{\mathrm{d}, \ell} \rightarrow J^{r}\left(\mathrm{R}, \mathrm{R}^{k}\right)
$$

by

$$
L_{\mathrm{d}, \ell}\left(j^{r} i(q)\right)=j_{1}^{r} F^{\mathrm{d}, \ell}(i(q)) .
$$

Then $\mathcal{S}_{\mathrm{d}, \ell}=L_{\mathbf{d}, \ell}^{-1}(\mathcal{S})$ is a Whitney stratification of $\mathcal{R}_{\mathrm{d}, \ell}$.

Let $i: Q \rightarrow M$ be an embedding with $i(Q) \cap \Sigma_{X}=\emptyset$. It is easy to show that $j^{r} i$ is transverse to $\mathcal{S}_{\mathrm{d}, \ell}$ on $\mathcal{R}_{\mathrm{d}, \ell}$ near $q \in Q$ if and only if $j_{1}^{r} F^{\mathrm{d}, \ell}$ is transverse to $\mathcal{S}$ near $i(q)$.

Define $T_{\mathrm{d}, \ell}$ as the set of embeddings $i: Q \rightarrow M$ such that $j^{r} i$ is transverse to $\mathcal{S}_{\mathrm{d}, \ell}$ on $\mathcal{R}_{\mathrm{d}, \ell}$. Since $\mathcal{S}_{\mathrm{d}, \ell}$ is a Whitney stratification, $T_{\mathrm{d}, \ell}$ is an open dense subset of $\operatorname{Emb}(Q, M)$. 
Because the choice of local equations depends only on the $\mathcal{C}$-equivalence, the set

$$
\mathcal{O}=\mathcal{O}_{1} \cap\left(\bigcap_{\mathbf{d}, \ell} T_{\mathrm{d}, \ell}\right)
$$

has required properties.

\section{Local properties of submanifolds}

In order to detect normal forms of vector fields near a generic submanifold, we shall study some properties of local equations. Denote by $\mathcal{E}_{n}$ the ring of function germs of $n$-variables at 0 and by $\mathfrak{m}_{n}$ the unique maximal ideal.

Let $F, G:\left(\mathbf{R} \times \mathbf{R}^{\ell}, 0\right) \rightarrow\left(\mathbf{R}^{k}, 0\right)$ be map germs. We say that $F$ and $G$ are $p$ - $\mathcal{C}^{+}$equivalent if there exists a diffeomorphism germ $\Phi:\left(R \times R^{\ell}, 0\right) \rightarrow\left(R \times R^{\ell}, 0\right)$ of the form $\Phi(x, u)=(x+\alpha(u), \phi(u))$ such that $\Phi^{*} I(F)=I(G)$, where $I(F)=F^{*}\left(\mathfrak{m}_{k}\right) \mathcal{E}_{1+\ell}$.

We remark that diffeomorphism germs of the above form preserve the vector field $\partial / \partial x$. The purpose of this section is to classify local equations by the $p-\mathcal{C}^{+}$-equivalence. For this purpose, we use some notations and results in [3], [4] and [5].

For each map-germ $f:(\mathrm{R}, 0) \rightarrow\left(\mathrm{R}^{k}, 0\right)$, we set

and

$$
T_{e} \mathcal{K}(f)=\left\langle\frac{\partial f}{\partial x}\right)_{\mathcal{E}_{1}}+I(f) \mathcal{E}_{1, k}
$$

$$
\mathcal{K}-\operatorname{cod}(f)=\operatorname{dim}_{\mathbf{R}} \mathcal{E}_{1, k} / T_{e} \mathcal{K}(f)
$$

where $\mathcal{E}_{1, k}$ is the $\mathcal{E}_{1}$-module of map germs $(\mathrm{R}, 0) \rightarrow \mathbf{R}^{k}$. Then we have the following well-known classification (cf.[3]).

Lemma 2.1. Let $f:(\mathbf{R}, 0) \rightarrow\left(\mathbf{R}^{k}, 0\right)$ be a map-germ with $\mathcal{K}$-cod $(f)<+\infty$. Then $f$ is $\mathcal{C}$-equivalent to the map-germ $\left(x^{t+1}, 0, \ldots, 0\right)$ for some non-negative integer $t$.

By the direct calculation, we have

$$
\mathcal{K}-\operatorname{cod}\left(x^{t+1}, 0, \ldots, 0\right)=(t+1) k-1 .
$$

The following notion is rather an important chain of ideas: A map germ $F:\left(\mathbf{R} \times \mathbf{R}^{\ell}, 0\right) \rightarrow\left(\mathbb{R}^{k}, 0\right)$ is an infinitesimally $\mathcal{C}^{+}$-versal deformation of $f=F \mid \mathbf{R} \times 0$ if

$$
\mathcal{E}_{1, k}=\left\langle\frac{d f}{d x}\right\rangle_{\mathbf{R}}+I(f) \mathcal{E}_{1, k}+\left\langle\frac{\partial F}{\partial u_{1}}\left|\mathbf{R} \times 0, \ldots, \frac{\partial F}{\partial u_{\ell}}\right| \mathbf{R} \times 0\right\rangle_{\mathbf{R}} .
$$

Then we have the following theorem, which is a corollary of Damon's general versality theorem in [2]. 
ThEoREM 2.2. Let $F, G:\left(\mathbf{R} \times \mathbf{R}^{\ell}, 0\right) \rightarrow\left(\mathbf{R}^{k}, 0\right)$ be infinitesimally $\mathcal{C}^{+}$-versal deformations of $f$. Then $F$ and $G$ are $p-\mathcal{C}^{+}$-equivalent.

Since $f$ is a map-germ of one-variable, we have

$$
\left\langle\frac{d f}{d x}\right\rangle_{\mathbf{R}}+I(f) \mathcal{E}_{1, k}=\left\langle\frac{d f}{d x}\right\rangle_{\mathcal{E}_{1}}+I(f) \mathcal{E}_{1, k} .
$$

Thus it follows that $F$ is an infinitesimally $\mathcal{C}^{+}$-versal deformation if and only if it is infinitesimally $\mathcal{K}$-versal. Hence the following classification theorem holds as a corollary of Theorem 2.2.

CoRollary 2.3. Let $F, G:\left(\mathbf{R} \times \mathbf{R}^{\ell}, 0\right) \rightarrow\left(\mathbf{R}^{k}, 0\right)$ be infinitesimally $\mathcal{K}$-versal deformations of $f, g:(\mathbf{R}, 0) \rightarrow\left(\mathbf{R}^{k}, 0\right)$ respectively. Then $F$ and $G$ are $p-\mathcal{C}^{+}$-equivalent if and only if $f$ and $g$ are $\mathcal{C}$-equivalent.

By Lemma 2.1 and Corollary 2.3, we can detect normal forms of local equations.

Propositron 2.4. Let $F:\left(\mathbf{R} \times \mathbf{R}^{n-1}, 0\right) \rightarrow\left(\mathbf{R}^{k}, 0\right)$ be an infinitesimally $\mathcal{K}$-versal deformation of $f:(\mathbf{R}, 0) \rightarrow\left(\mathbb{R}^{k}, 0\right)$. Then $F$ is $p-\mathcal{C}^{+}$-equivalent to

$(*) \quad\left(x^{t+1}+\sum_{i=1}^{t} u_{1, i} x^{t-i}, \sum_{i=1}^{t+1} u_{2, i} x^{t+1-i}, \ldots, \sum_{i=1}^{t+1} u_{k, i} x^{t+1-i}\right)$

for some integer $t,\left(0 \leq t \leq \frac{n}{k}-1\right)$. Here,

$$
(x, u)=\left(x, u_{1,1}, \ldots, u_{1, t}, u_{2,1}, \ldots, u_{2, t+1}, \ldots, u_{k, 1}, \ldots, u_{k, t+1}, u_{(t+1) k+1}, \ldots, u_{n}\right) .
$$

Proof: By the assumption and Lemma 2.1, $f$ is $\mathcal{C}$-equivalent to $\left(x^{t+1}, 0, \ldots, 0\right)$ for some integer $t$. Then $\mathcal{K}$-cod $(f)=(t+1) k-1$ and $0 \leq t \leq \frac{n}{k}-1$. By a simple calculation, the $(n+1)$-parameter infinitesimally $\mathcal{K}$-versal deformation of $\left(x^{t+1}, 0, \ldots, 0\right)$ is given by $\left(^{*}\right)$. It follows from Corollary 2.3 that $F$ is $p$ - $\mathcal{C}^{+}$-equivalent to $\left(^{*}\right)$. 


\section{Proof of Theorem A}

We now define a Whitney stratification $\mathcal{S}$ of $J^{r}(1, k)$ as follows:

$$
\mathcal{S}=\left\{0, \mathcal{K}\left(j^{r}\left(x^{t+1}, 0, \ldots, 0\right)\right) \mid 0 \leq t \leq r-1\right\}
$$

where $o=j^{r}(0, \ldots, 0)$ and $\mathcal{K} z$ denotes the $\mathcal{K}$-orbit through a jet $z$. Let $F:\left(\mathbf{R} \times \mathbf{R}^{n-1}, 0\right) \rightarrow$ $\left(\mathbf{R}^{k}, 0\right)$ be a map germ such that $j_{1}^{r} F$ is transverse to $\mathbf{R} \times\{0\} \times \mathcal{K}\left(j^{r}\left(x^{t+1}, 0, \ldots, 0\right)\right)$ for $r>\frac{n}{k}-1$. By the usual calculation, $F$ is an infinitesimally $\mathcal{K}$-versal deformation of $F \mid \mathbf{R} \times 0$. Then $F$ is $p$ - $\mathcal{C}^{+}$-equivalent to a map germ $\left({ }^{*}\right)$ in Proposition 2.4 .

We adopt a coordinate transformation of $\left(R \times R^{n-1}, 0\right)$ as follows:

$$
\begin{aligned}
x_{\ell k+1} & =\frac{1}{(t+1-\ell) !} x^{t+1-\ell}+\sum_{i=1}^{t-\ell} \frac{(t-i) !}{(t+1) !(t-i+\ell) !} u_{1, i} x^{t-i-\ell}, \quad(0 \leq \ell \leq t), \\
x_{\ell k+j} & =\sum_{i=1}^{t+1-\ell} \frac{(t-i+1) !}{(t-i+1-\ell) !} u_{j, i} x^{t-i+1-\ell}, \quad(0 \leq \ell \leq t, 2 \leq j \leq k), \\
x_{m} & =u_{m}, \quad((t+1) k+1 \leq m \leq n) .
\end{aligned}
$$

By this coordinate transformation, the zero set of the germ $\left(^{*}\right)$ in Proposition 2.4 is diffeomorphic to the set $\left\{x_{1}=\cdots=x_{k}=0\right\}$, and the vector field $\partial / \partial x$ is transformed to

$$
\sum_{j=1}^{t}\left(\sum_{i=1}^{k} x_{j k+i} \frac{\partial}{\partial x_{(j-1) k+i}}\right)+\frac{\partial}{\partial x_{t k+1}}
$$

By Theorem 1.1, the set of embeddings $Q \rightarrow M$ satisfying the property in Theorem A is residual. This completes the proof. 


\section{Some general cases}

In this section we consider more general situations which include two cases. One of these cases is that $i(Q)$ is a generic hypersurface with boundary in $M$ and another case is that $i(Q)$ is a generic piecewise smooth hypersurface. We now divide these two cases.

i) Hypersurface with boundary: In this case the local equation of $i(Q)$ at a boundary point $i(q)$ is given by a local submersion $\left(F_{1}, F_{2}\right):(M, i(q)) \rightarrow\left(\mathbf{R}^{2}, 0\right)$ such that $F_{1}^{-1}(0) \cap\left\{F_{2} \geq 0\right\}=(i(Q), i(q))$ and $F_{1}^{-1}(0) \cap F_{2}^{-1}(0)=(i(\partial Q), i(q))$. Then we use the following equivalence relation: Let $\left(F_{1}, F_{2}\right),\left(G_{1}, G_{2}\right):\left(\mathbf{R} \times \mathbf{R}^{\ell}, 0\right) \rightarrow\left(\mathbf{R}^{2}, 0\right)$ be map germs. We say that $\left(F_{1}, F_{2}\right)$ and $\left(G_{1}, G_{2}\right)$ are $p-\left(\mathcal{B}, \mathcal{C}^{+}\right)$-equivalent if there exists a diffeomorphism germ $\Phi:\left(\mathbf{R} \times \mathbf{R}^{\ell}, 0\right) \rightarrow\left(\mathbf{R} \times \mathbf{R}^{\ell}, 0\right)$ of the form $\Phi(x, u)=(x+\alpha(u), \phi(u))$ such that

$$
A \cdot\left(\begin{array}{l}
\Phi^{*}\left(F_{1}\right) \\
\Phi^{*}\left(F_{2}\right)
\end{array}\right)=\left(\begin{array}{l}
G_{1} \\
G_{2}
\end{array}\right)
$$

for some $A \in \Delta_{+}\left(2, \mathcal{E}_{1+\ell}\right)$. Here

$$
\Delta_{+}\left(2, \mathcal{E}_{1+\ell}\right)=\left\{\left(\begin{array}{cc}
\xi_{11} & 0 \\
\xi_{21} & \xi_{22}
\end{array}\right) \mid \xi_{i j} \in \mathcal{E}_{1+\ell}, \xi_{11} \cdot \xi_{22} \neq 0 \text { and } \xi_{22}>0\right\} .
$$

We can classify generic local equations by the $p-\left(\mathcal{B}, \mathcal{C}^{+}\right)$-equivalence almost the same way as that of Proposition 2.4. After that we also transform local equations and vector field $\partial / \partial x$ to normal forms in the following theorem:

THEOREM B. Local normal forms of vector fields at a boundary point of a generic hypersurface with boundary are given by

$$
\sum_{j=1}^{r}\left(x_{2 j+1} \frac{\partial}{\partial x_{2 j-1}}+x_{2 j+2} \frac{\partial}{\partial x_{2 j}}\right)+\frac{\partial}{\partial x_{2 r+1}}+\sum_{i=0}^{s-r-1} x_{2(r+1)+i+1} \frac{\partial}{\partial x_{2(r+1)+i}}
$$

for some pairs of integers $(r, s)$ with $0 \leq r \leq \frac{n-4}{2}$ and $r+1 \leq s \leq \frac{n}{2}$, where the hypersurface is locally given by

$$
\left\{\left(x_{1}, \ldots, x_{n}\right) \mid x_{1}=0 \text { and } x_{2} \geq 0\right\} .
$$

ii) Piecewise-smooth hypersurface: In what follows, we shall take as a local model of piecewise-smooth hypersurface the germ at the origin of sets

$$
\begin{aligned}
& \quad Q_{k}=\bigcup_{i=1}^{k}\left\{\left(x_{1}, \ldots, x_{n}\right) \mid x_{1} \geq 0, x_{2} \geq 0, \ldots, x_{i-1} \geq 0, x_{i}=0, x_{i+1} \geq 0, \ldots, x_{k} \geq 0\right\}, \\
& (k=1, \ldots, n) .
\end{aligned}
$$


Any piecewise-smooth hypersurface is locally the image of $Q_{k}$ by a diffeomorphism germ $i:\left(\mathbf{R}^{n}, 0\right) \rightarrow(M, q)$ for some $k$. In this case the local equation is given by a local submersion $F=\left(F_{1}, \ldots, F_{k}\right):(M, q) \rightarrow\left(\mathrm{R}^{k}, 0\right)$ such that

$$
\bigcup_{i=1}^{k}\left(F_{i}^{-1}(0) \cup\left\{F_{j} \geq 0 \mid 1 \leq j \leq k, j \neq i\right\}\right)=\left(i\left(Q_{k}\right), q\right) .
$$

Then we use the following equivalence relation: Let $F, G:\left(\mathbf{R} \times \mathbf{R}^{\ell}, 0\right) \rightarrow\left(\mathbf{R}^{k}, 0\right)$ be mapgerms. We say that $F$ and $G$ are $p-\left(Q_{k}, \mathcal{C}^{+}\right)$-equivalent if there exists a diffeomorphism germ $\Phi:\left(\mathbf{R} \times \mathbf{R}^{\ell}, 0\right) \rightarrow\left(\mathbf{R} \times \mathbf{R}^{\ell}, 0\right)$ of the form $\Phi(x, u)=(x+\alpha(u), \phi(u))$ such that

for some $A \in D_{+}\left(k, \mathcal{E}_{1+\ell}\right)$. Here,

$$
A \cdot\left(\begin{array}{c}
\Phi^{*}\left(F_{1}\right) \\
\vdots \\
\Phi^{*}\left(F_{k}\right)
\end{array}\right)=\left(\begin{array}{c}
G_{1} \\
\vdots \\
G_{k}
\end{array}\right)
$$

$$
D_{+}\left(k, \mathcal{E}_{1+\ell}\right)=\left\{\left(\begin{array}{ccc}
\xi_{\mathrm{i}} & & 0 \\
& \ddots & \\
0 & & \xi_{k}
\end{array}\right) \mid \xi_{i} \in \mathcal{E}_{1+\ell}, \xi_{i}>0\right\}
$$

Similarly to Theorems A and B, we have the following classification:

THEOREM C. Local normal forms of generic vector fields at a point of a generic piecewisesmooth hypersurface are labelled by a positive integer $k$ and integers $t_{i}, 1 \leq i \leq k$, with

and given by

$$
\sum_{i=1}^{k} t_{i} \leq n \text { and } t_{1} \geq t_{2} \geq \cdots \geq t_{k} \geq 1
$$

$$
\begin{gathered}
\sum_{i=1}^{m}\left(x_{t_{1}+\cdots+t_{i-1}+k+2-i} \partial / \partial x_{i}+\sum_{j=1}^{t_{i}-2} x_{t_{1}+\cdots+t_{i-1}+k+2-i+j} \partial / \partial x_{t_{1}+\cdots+t_{i-1}+k+1-i+j}\right. \\
\left.+\partial / \partial x_{t_{1}+\cdots+t_{i}+k-i}\right)+\sum_{i=m+1}^{k}\left( \pm \partial / \partial x_{i}\right),
\end{gathered}
$$

and the piecewise-smooth hypersurface is locally given by $Q_{k}$, where $m$ is an integer with $t_{m} \geq 2, t_{m+1}=1$.

EXAMPLE 4.1: Applying Theorem $\mathrm{C}$ to the case $n=3$ and $k \geq 2$, we have the local normal forms of generic vector fields at a non-smooth point of a generic piecewise-smooth surface in $\mathrm{R}^{3}$ :

$$
\begin{gathered}
\pm \frac{\partial}{\partial x_{1}} \pm \frac{\partial}{\partial x_{2}} \pm \frac{\partial}{\partial x_{3}}, \quad \text { at the summit of } Q_{3} \\
x_{3} \frac{\partial}{\partial x_{1}} \pm \frac{\partial}{\partial x_{2}}+\frac{\partial}{\partial x_{3}}, \pm \frac{\partial}{\partial x_{1}} \pm \frac{\partial}{\partial x_{2}}, \quad \text { at the edge of } Q_{2}
\end{gathered}
$$




\section{REFERENCES}

1. Arnol'd, V.I., On local problems of analysis, Vestnik Moscov Gos. Univ. Ser. Mat. Mekh. 25-2 (1970), 52-55.

2. Damon, J., The unfolding and determinacy theorems for subgroups of $\mathcal{A}$ and $\mathcal{K}$, Memoirs of Amer. Math. Soc. 50-306 (1984).

3. Gibson, C.G., "Singular Points of Smooth Mappings," Pitman, 1979.

4. Martinet, J., "Singularities of Smooth Functions and Maps," Cambridge Univ. Press, 1982.

5. Mather, J., Stability of $C^{\infty}$ mappings III: finitely determined map-germs, Publ. Math. I.H.E.S. 35 (1969), 127-156.

6. Vishik, S.M., Vector fields near the boundary of a manifold, Vestnik Moscov Gos. Univ. Ser. Mat. Mekh. 27-1 (1972), 21-28.

7. Watanabe, K., Master thesis (in Japanese), Hokkaido University (1991).

Department of Mathematics, Hokkaido University, Sapporo 060, Japan. 